\title{
Bone Marrow Necrosis and Fat Embolism in a Patient with Sickle Cell Crisis, a Fatal Complication
}

\author{
Gagandeep Kaur*, Oluwaseyi Olayinka and Gunjan Gupta \\ Department of Pathology and Laboratory Medicine, Danbury Hospital, Danbury, Connecticut, USA

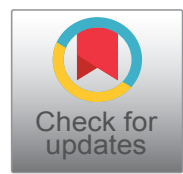

*Corresponding author: Gagandeep Kaur, MD, Department of Pathology and Laboratory Medicine, Danbury Hospital, Danbury, Connecticut, USA, Tel: 203-300-9148

\begin{abstract}
Sickle cell disease is a group of inherited disorder due to substitution of valine for glutamic acid at position 6 of beta globin chain of hemoglobin [1] leading to sickling of red blood cells. It is most common in African ancestry. The sickled RBC's can cause vaso-occlusive crisis leading to dreadful complications. We report a case of a 56-year-old female with past medical history of sickle cell disease, atrial fibrillation and prior history of stroke. She presented to the emergency department with chief complaint of left leg pain. After few hours the pain moved to the lower and upper back. Accompanying symptoms included shortness of breath, nausea and vomiting. Laboratory findings were significant for mild lactic acidosis, elevated LDH, hyperbilirubinemia and leukocytosis. She was started with opioids and intravenous fluids for sickle cell crisis. Unfortunately, overnight, the patient expired. On autopsy, the cause of death was found to be bone marrow necrosis and bone marrow and fat embolism to lungs causing fatal impairment of lung function.
\end{abstract}

\section{Keywords}

Sickle cell crisis, Bone marrow, Fat embolism

\section{Introduction}

Sickle cell disease (SCD) is a genetic disorder characterized by the presence of a mutated form of hemoglobin, hemoglobin S (HbS). The disorder includes all genotypes containing at least one sickle gene, in which $\mathrm{HbS}$ makes up at least half the hemoglobin present [1]. HbSS, also known as sickle cell anemia, is the most common and most severe form of the disease. Other forms of the disease include $\mathrm{HbSC}, \mathrm{HbS}$ beta thalassemia, HbSD, HbSE, and HbSO [2]. Patients with SCD commonly present with features of vascular occlusion, a phenomenon that lead to life threatening events including fat embolism (FE). FE is a rare but fatal complication of SCD secondary to extensive bone marrow infarction and necrosis. The condition was first described as a complication of SCD

more than five decades ago in a patient with generalized body pain [3]. Since then several cases have been reported in the literature. However, in many of these cases, the diagnosis of FE was not made until post-mortem examination indicating that the condition is still not widely recognized [4]. This finding underscores the need for continued reporting of cases of FE as awareness and early recognition of this fatal complication can lower morbidity and mortality in SCD. We report autopsy findings in a 56-year-old female with HbSS who presented in vaso-occlusive crisis with subsequent bone marrow necrosis and FE.

\section{Case Description}

The deceased was a 56-year-old female with past medical history of sickle cell disease (HbSS), atrial fibrillation, stroke, hypertension, hypothyroidism and sickle cell crisis. She was diagnosed with sickle cell disease 20 years ago. She presented with pain in the left leg of few hours duration, which later moved to lower and upper back. Other complaint was shortness of breath without exertion. She denied cough and chest pain. Laboratory work up showed increased WBC- $22.9 \times 10(9) / L$, increased RBC- $5.37 \times 10(12) / L$, high lactic acid- $2.3 \mathrm{mmol} / \mathrm{IL}$ and high LDH- $817 \mathrm{U} / \mathrm{L}$. Chest X-Ray showed clear lungs. Unfortunately overnight, the patient was found to be unresponsive and

Citation: Kaur G, Olayinka O, Gupta G (2019) Bone Marrow Necrosis and Fat Embolism in a Patient with Sickle Cell Crisis, a Fatal Complication. Int J Pathol Clin Res 5:104. doi.org/10.23937/24695807/1510104

Accepted: November 20, 2019: Published: November 22, 2019

Copyright: (c) 2019 Kaur G, et al. This is an open-access article distributed under the terms of the Creative Commons Attribution License, which permits unrestricted use, distribution, and reproduction in any medium, provided the original author and source are credited. 

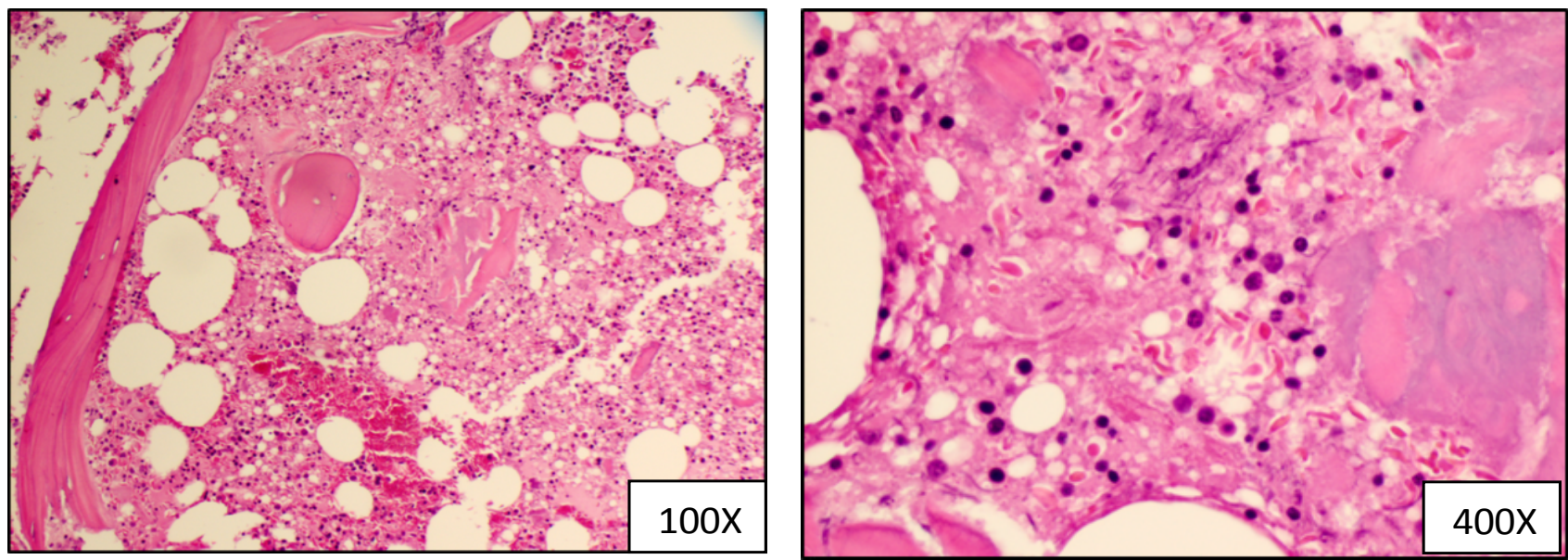

Figure 1: Hematoxylin and eosin staining demonstrating bone marrow necrosis.
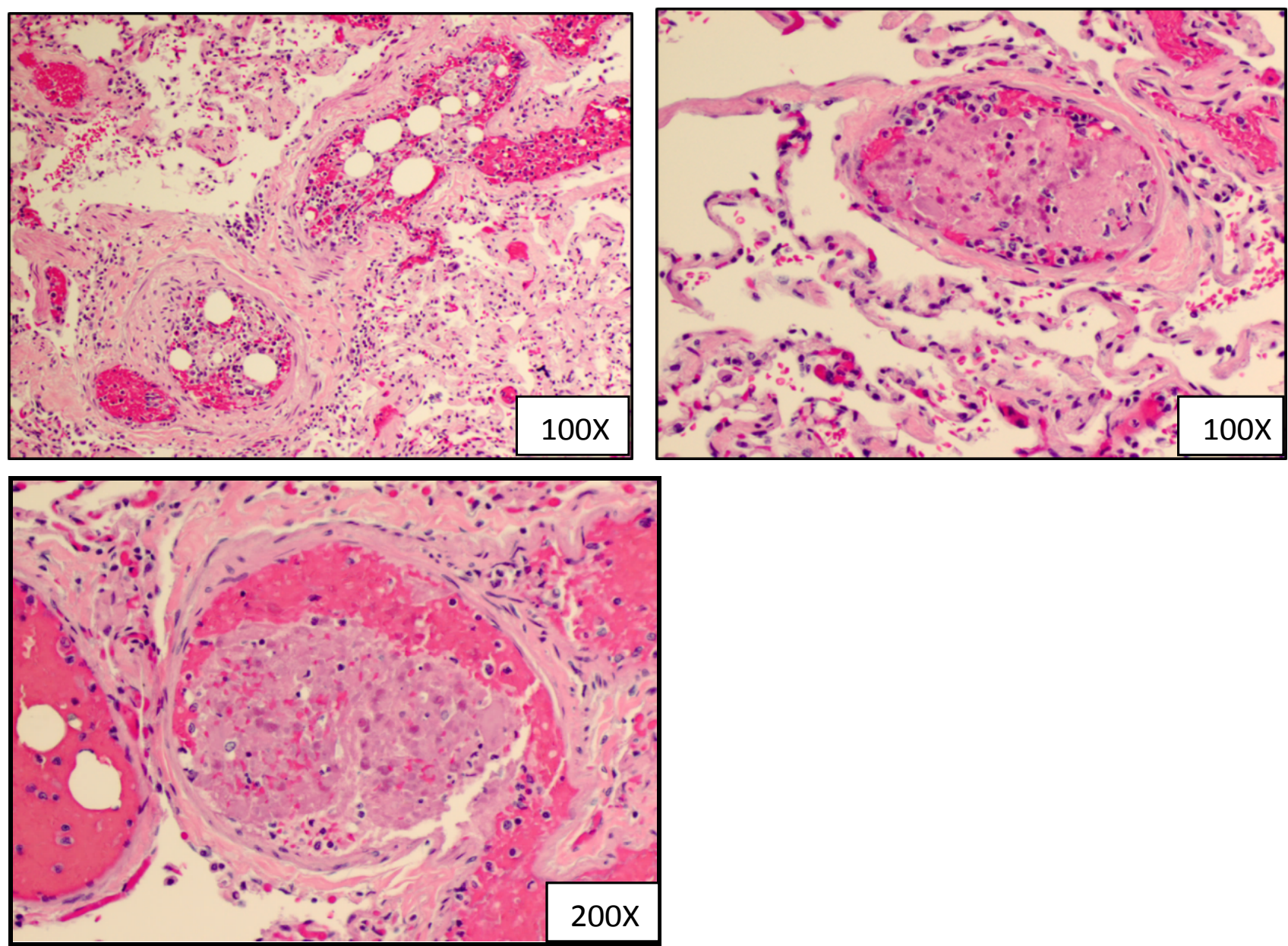

Figure 2: Hematoxylin and eosin staining demonstrating fat and bone marrow embolism in the pulmonary vasculature.

pulseless. She was pronounced dead and an autopsy was performed. Significant gross examination findings included firm and atrophic spleen, congested lungs, hepatomegaly, two small hemorrhagic areas in the right basal ganglion and cardiomegaly with septal and left ventricular hypertrophy. Upon microscopic examination marked vascular congestion with sickled RBC's was seen in the multiple organs including lungs, liver, kidneys, brain, spleen, pituitary gland and bone marrow. Sections of the thoracic and lum- bar vertebra revealed areas of hemorrhage, sickled red blood cells and areas of bone marrow necrosis (Figure 1). Examination of the lungs revealed marked vascular congestion and necrotic bone marrow and fat embolism in branches of the pulmonary artery, arterioles and alveolar septal capillaries (Figure 2). The brain showed two recent hemorrhagic infarctions in the basal ganglia and a small thromboembolus in the left temporal lobe. (Figure 3 and Figure 4) The spleen was firm atrophic, calcified with fibrosis, congestion 

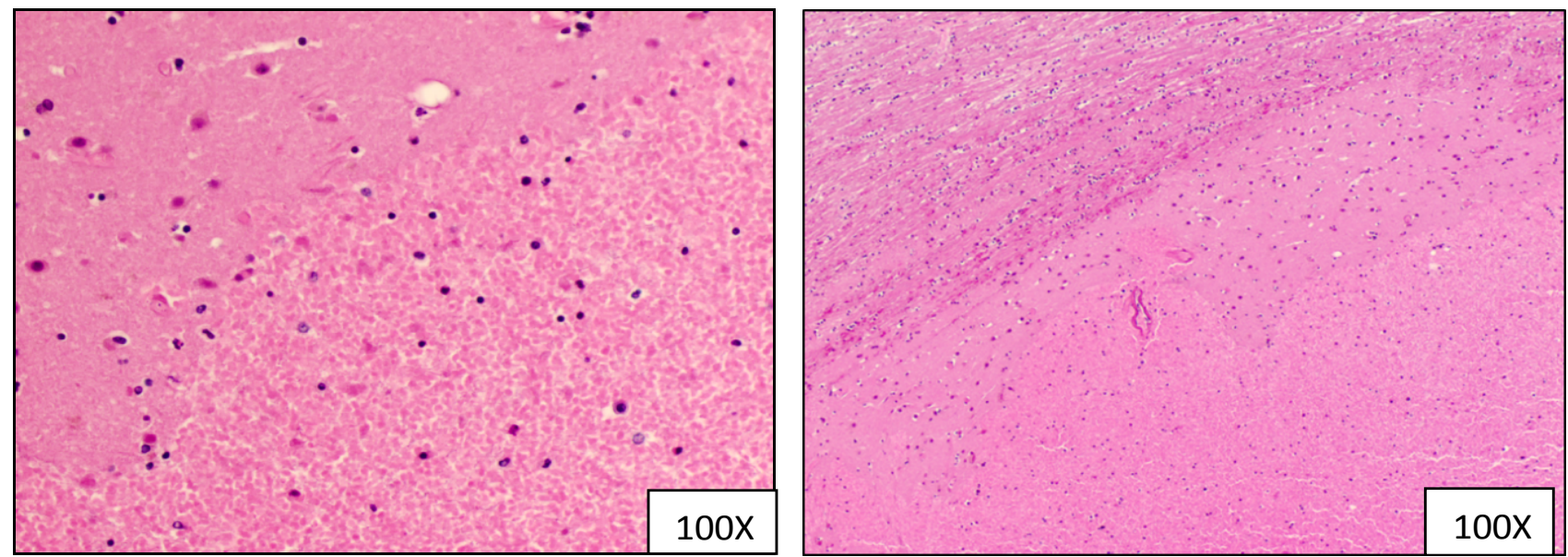

Figure 3: Hematoxylin and eosin staining demonstrating two hemorrhagic infarcts in the basal ganglia.
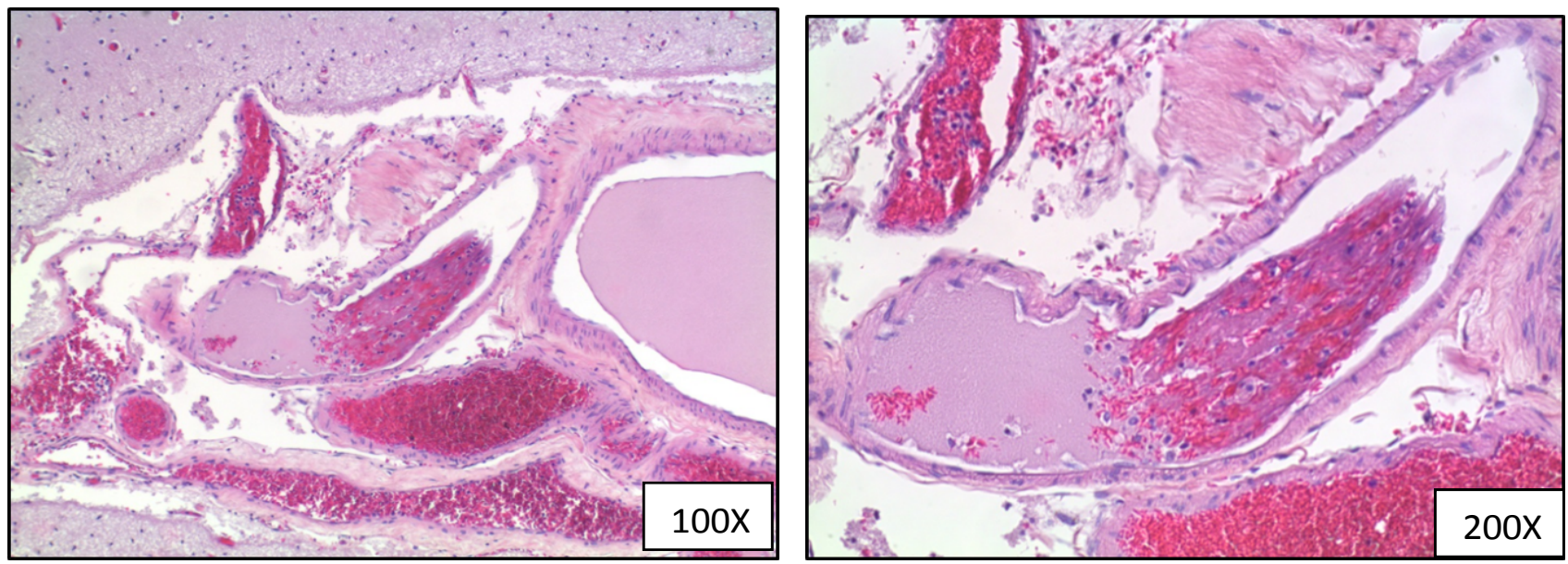

Figure 4: Hematoxylin and eosin staining demonstrating thromboembolus in the left temporal lobe.

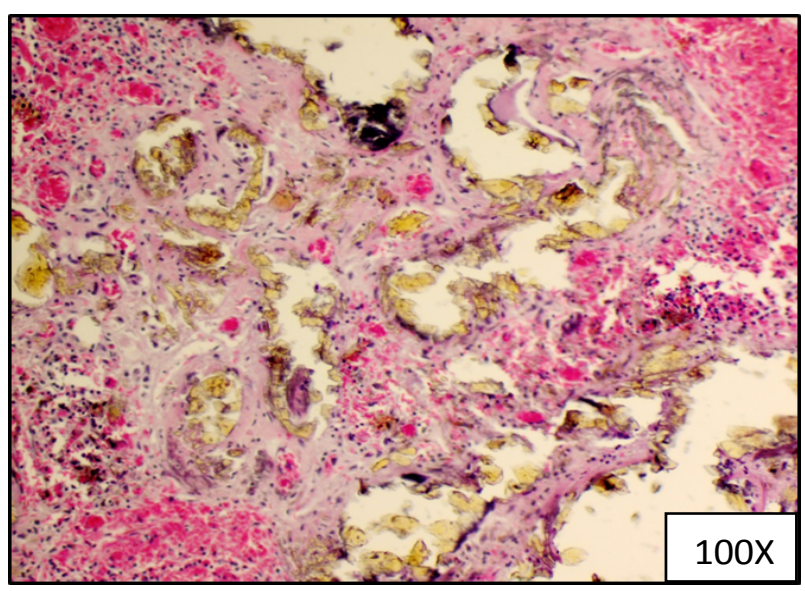

Figure 5: Hematoxylin and eosin staining showing Gamma-Gandy bodies in the spleen.

and numerous Gamma-Gandy bodies (Figure 5). Both kidneys showed acute tubular necrosis. Other significant findings included cardiomegaly (450 grams), left ventricular hypertrophy $(1.6 \mathrm{~cm})$ and hypertrophy of the interventricular septum $(1.9 \mathrm{~cm})$ with two old myocardial infarctions and mild to moderate atherosclerosis of coronary arteries.

\section{Conclusions}

Fat embolism has emerged as an important complication of sickle cell disease and is associated with high morbidity and mortality. Although the pathogenesis remains unclear, fat embolism secondary to bone marrow necrosis is believed to result from passage of bone marrow material into circulation through bony venous channels. This process is facilitated by the porousness of cancellous bone containing red marrow [4]. Although the diagnosis of fat and bone marrow embolism is mostly seen in patients with trauma or fractured bones, it has also been reported in patients with sickle cell disease. Precipitating factors in these patients are dehydration, toxins, and infections. We are reporting a case of fat and bone marrow embolism in a patient with sickle cell crisis who came to the emergency room in acute pain and expired overnight. Although there have been a number of reported cases in literature, survival is low which indicates that awareness of this entity is low [4]. Key elements of a positive outcome are timely recognition, diagnosis and aggressive treatment. Suspicion should be high while taking care of sickle cell crisis patients with multiple organ involvement to provide appropriate medical care [5]. 


\section{References}

1. Joseph E Maakaron (2019) Sickle Cell Anemia. Medscape.

2. https://www.cdc.gov/ncbddd/sicklecell/documents/ SCD-factsheet_What-is-SCD.pdf

3. Wade LJ, Stevenson LD (1941) Necrosis of the bone marrow with fat embolism in sickle cell anemia. Am J Pathol 17: 47-54.
4. Dang NC, Johnson C, Eslami-Farsani M, Haywood LJ (2005) Bone marrow embolism in sickle cell disease: A review. American Journal of Hematology 79: 61-67.

5. Susan Shyu, Mario Rubin, Cinthia B Drachenberg (2017) Fatal unsuspected fat embolism syndrome in a sickle cell patient. Human Pathology: Case Reports 8: 62-64. 